\title{
Diseño de una rúbrica para la obtención de criterios de evaluación de los Trabajos Final de Grado de la Escuela Técnica Superior de Ingeniería Agronómica y del Medio Natural
}

\author{
Mercedes Verdeguer Sanchoa, Juan Antonio Llorens Molinab, Francisco García \\ Breijo $^{a}$, Josefa Roselló Caselles ${ }^{a}$, Alberto García Prats ${ }^{c}$, Constanza Rubio Michavilad, \\ María Vargas Colás ${ }^{\mathrm{e}}$, María Pilar Santamarina Siurana ${ }^{a}$ \\ a(Departamento de Ecosistemas Agroforestales (DEPEAF), Escuela Técnica Superior de Ingeniería \\ Agronómica y del Medio Natural (ETSIAMN), Universitat Politécnica de València (UPV), \\ merversa@doctor.upv.es, fjgarci@eaf.upv.es, jrosello@upvnet.upv.es, mpsantam@eaf.upv.es) \\ b(Departamento de Química, ETSIAMN, UPV, juallom2@qim.upv.es) \\ c(Departamento de Ingeniería Hidráulica y Medio Ambiente, agprats@upvnet.upv.es) \\ d(Departamento de Física Aplicada, crubiom@fis.upv.es) \\ e(Departamento de Tecnología de Alimentos, mavarco@tal.upv.es)
}

\begin{abstract}
The present paper includes some results obtained in the context of the teaching innovation project "Tools for the planning, realization and assesment of the Bachelor's Degree Thesis (BDT)". The project aims to solve the problems that students, advisors and members of the evaluation board have to face in terms of the assesment of the BDT, providing evaluation tools (rubrics). At present, there are no clear and objective criteria for assessing the BDTs, and their evaluation sometimes is submitted to the subjective assessment of the members of the board. What is more, at the moment the formulary that is used to evaluate the transversal UPV outcomes is more complete and detailed than the one used for the evaluation of the BDT. Therefore, it is necessary to develop a document named as "Bachelor's Degree Thesis Guide", which will provide tools for an adequate and optimal planning and evaluation of BDTs. One of the key points of the project has been the elaboration of a rubric with measurable indicators that facilitate and clarify the evaluation of the BDTs. This rubric will be distributed among the different interested groups (professors, students and academic boards) to improve and validate it.
\end{abstract}

Keywords: evaluation, assessment, criteria, rubric

\section{Resumen}

El presente trabajo recoge parte de los resultados obtenidos en el marco del proyecto de innovación docente "Herramientas para la planificación, realización y valoración de los Trabajo Fin de Grado (TFGs)". Se pretende resolver la problemática a la que se enfrenta el alumnado, los tutores y los tribunales en cuanto a los criterios de valoración de los TFGs, aportando herramientas para su evaluación (rúbricas). Actualmente, no existen unos 
criterios claros y objetivos para la evaluación de los TFGs, quedando en ocasiones su calificación sometida a valoraciones subjetivas, por parte del tribunal evaluador. Así, es más detallado el documento para la evaluación de las competencias trasversales que el utilizado para la evaluación del TFG. Por tanto, es necesario desarrollar un documento "Guía del Trabajo Fin de Grado", que proporcione instrumentos para una adecuada y óptima planificación y evaluación de los TFGs. Uno de los puntos clave del proyecto, ha sido la elaboración de una rúbrica con indicadores medibles que faciliten y clarifiquen la evaluaciónn de los TFGs. Dicha rúbrica, se pasará a los diferentes grupos de interés (profesorado, alumnado y comisiones académicas) para mejorarla y validarla.

Palabras clave: evaluación, valoración, criterios, rúbrica

\section{Introducción}

Los Trabajos Fin de Grado, en adelante (TFGs), constituyen un trabajo de naturaleza profesional original en el que quedan de manifiesto los conocimientos, habilidades y competencias adquiridas por el estudiante a lo largo de sus estudios de Grado. Los tutores del TFG determinarán cuáles son los contenidos específicos del TFG, de acuerdo con las temáticas recogidas en la normativa correspondiente:

(http://www.upv.es/entidades/ETSIAMN/info/Normativa_Trabajo_Final1.pdf).

Actualmente existe cierta dificultad a la hora de planificar, realizar el seguimiento, y evaluar los TFGs, ya que no existe ninguna guía de referencia que regule estos aspectos, ya que los documentos que se han preparado hasta el momento se han centrado en aspectos formales y de contenidos mínimos. Además, en muchos trabajos no se estima adecuadamente la dedicación del alumno, que frecuentemente invierte para la realización del TFG muchas más horas de trabajo que las reflejadas en los créditos correspondientes (12 ECTS).

Los profesores tutores de los TFGs tienen que verificar durante la realización de los mismos que el alumno está llevando a cabo un desarrollo adecuado de las tareas correspondientes para alcanzar sus objetivos, de acuerdo con las competencias profesionales que debe trabajar durante su realización. No obstante, no se dispone actualmente de un documento donde se puedan recoger los progresos del alumno durante la realización del TFG y que permita al tutor del mismo verificar que se está ejecutando de forma adecuada.

Entre las estrategias habitualmente utilizadas para analizar las diferentes dimensiones y/o aspectos objeto de preocupación en torno a los principales agentes implicados en los procesos de elaboración, tutorización, presentación y/o defensa y evaluación de los TFGs, se encuentran las encuestas de opinión y las entrevistas al alumnado y a los tutores (Webster, et al., 2000). Además, otro de los aspectos críticos es la evaluación de los TFGs, cada vez más azarosa, pues al no disponer de unos criterios objetivos claros y establecidos, el alumnado puede percibir que se le está evaluando de manera subjetiva. Actualmente no

(cc) EY-NC-ND 2017, Universitat Politècnica de València

Congreso IN-RED (2017) 
Mercedes Verdeguer Sancho, Juan Antonio Llorens Molina, Francisco García Breijo, Josefa Roselló Caselles, Alberto García Prats, Constanza Rubio Michavila, María Vargas Colás, María Pilar Santamarina Siurana

se dispone de un documento de referencia (rúbrica), donde se recojan de forma clara y objetiva, los criterios que va a tener en cuenta el tribunal para evaluar el TFG en los diferentes Grados de la ETSIAMN. En dicha rúbrica, deberán recogerse los indicadores a las acciones de evaluación, así como exactamente el nivel de cumplimiento que se le exige al alumnado en cada indicador para que la evaluación sea lo más objetiva posible, independientemente de quién sea el agente evaluador (Valderrama et al., 2009).

Para ayudar a resolver esta problemática en la realización de los TFGs, varios profesores de la Escuela Técnica Superior de Ingeniería Agronómica y del Medio Natural planteamos un proyecto de Innovación Docente titulado "Herramientas para la planificación, realización y valoración de los Trabajos Fin de Grado". El equipo de trabajo del proyecto de innovación ha querido trabajar de la mano de la Dirección de la Escuela Técnica Superior de Ingeniería Agronómica y del Medio Natural. Por ello, está formado por la Jefa de Estudios de la Escuela, la Subdirectora de la Comisión Académica del Título Grado en Ingeniería Agroalimentaria y del Medio Rural, el Subdirector de la Comisión Académica del Título Grado en Ingeniería Forestal y del Medio Natural, y por cinco profesores de las áreas de química, biología, botánica y tecnología de alimentos, que imparten docencia tanto en el Grado como en los Masteres, entre ellos una Directora de Departamento.

El proyecto responde a la necesidad con la que se encuentran tanto el alumnado como los tutores y tribunales en relación a la planificación y, fundamentalmente, la evaluación del Trabajo Fin de Grado (TFG), el tema más prioritario en estos momentos, aportando una herramienta adecuada y objetiva, una rúbrica. Es imprescindible que el alumno conozca de antemano el sistema de evaluación utilizado para valorar su trabajo, al igual que sucede con las asignaturas que cursa.

Las rúbricas se han propuesto y utilizado como elementos para la tutorización y evaluación del aprendizaje de los alumnos en enseñanzas universitarias (Torres y Perea, 2010; GarcíaRos, 2011; Raposo y Martínez, 2011), pero es imprescindible elaborar rúbricas que cumplan con criterios de fiabilidad y validez (Berrocoso, 2014). Existe una experiencia previa en cuanto al uso de rúbricas para el seguimiento y evaluación de los Proyectos Fin de Carrera, llevada a cabo en la Escuela Superior Politécnica de la Universitat Pompeu Fabra (Moreno et al., 2012), cuyos primeros resultados, basados en informaciones provenientes tanto de directores, miembros de tribunal como de los propios estudiantes, muestran que, de manera global, el uso de rúbricas permite sistematizar tanto el seguimiento como la evaluación de los trabajo realizados, a la vez que facilita la homogenización de criterios (en clave de rigurosidad y transparencia). Otras Universidades, como la Universitat de les Illes Balears, también están desarrollando proyectos de innovación docente para elaborar rúbricas para la evaluación de TFGs y TFMs (Jaume-iCapó et al., 2012).

\section{Objetivos}

El objetivo de este trabajo es desarrollar un documento-rúbrica en el que se establezcan los criterios de valoración que se tendrán en cuenta a la hora de evaluar un TFG en la ETSIAMN. 
Para la consecución del mismo, se plantean los siguientes objetivos específicos:

1. Obtener información sobre la situación actual en relación a la valoración de los TFGs en la ETSIAMN, reunir la documentación utilizada para la evaluación de los mismos.

2. Analizar cómo se realiza la evaluación de TFGs en otras Escuelas de la UPV o en otras Universidades y reunir la documentación utilizada para la evaluación de los mismos.

3. Elaborar un documento (rúbrica) en el que se establezcan los criterios e indicadores de valoración que se tendrán en cuenta por el tribunal de calificación para evaluar los TFG, que deberá ser validado por los diferentes grupos de interés.

\section{Desarrollo de la innovación}

De acuerdo con los objetivos planteados, se procedió a recopilar la información actualmente disponible y se analizó la situación actual de los TFGs en diferentes Escuelas de la Universitat Politècnica de València, revisando las rúbricas e instrumentos de evaluación disponibles para la valoración de los TFGs. En una primera aproximación, se elaboró una primera versión de la rúbrica que fue mostrada en Comisiones Académicas para recoger los comentarios de los miembros de las mismas (Figura 1).

\begin{tabular}{|c|c|c|c|c|}
\hline \multicolumn{5}{|c|}{ Rúbrica para la evaluación del documento TFG y de la defensa } \\
\hline INDICADOR & NIVEL 1 & NIVEL 2 & NIVEL 3 & NIVEL 4 \\
\hline $\begin{array}{l}\text { Adecuación a objetivos } \\
\text { formativos y competencias de la } \\
\text { titulación }\end{array}$ & $\begin{array}{l}\text { No se adecúa a los } \\
\text { objetivos formativos }\end{array}$ & $\begin{array}{l}\text { Se adecúa de forma } \\
\text { parcial }\end{array}$ & $\begin{array}{l}\text { Se adecúa en gran medida } \\
\text { a los objetivos formativos }\end{array}$ & $\begin{array}{l}\text { Se adecúa de forma } \\
\text { completa a los objetivos } \\
\text { formativos }\end{array}$ \\
\hline Introducción y Objetivos & $\begin{array}{l}\text { No aparecen los objetivos } \\
\text { o se confunden con plan } \\
\text { de trabajo o metodología. }\end{array}$ & $\begin{array}{l}\text { Los objetivos no están } \\
\text { claros y no se justifica el } \\
\text { TFG. El problema a } \\
\text { resolver no está definido. }\end{array}$ & $\begin{array}{l}\text { No se especifican todos } \\
\text { los objetivos o no quedan } \\
\text { muy claros. La temática y } \\
\text { contribución se justifica }\end{array}$ & $\begin{array}{l}\text { Justifica la temática, } \\
\text { especifica todos los } \\
\text { objetivos y evidencia la } \\
\text { contribución original. }\end{array}$ \\
\hline $\begin{array}{l}\text { Antecedentes y reconocimiento } \\
\text { de trabajos previos ajenos. }\end{array}$ & No cita & $\begin{array}{l}\text { Cita otros trabajos sin } \\
\text { sistematización }\end{array}$ & $\begin{array}{l}\text { Cita otros trabajos de } \\
\text { forma sistemática }\end{array}$ & $\begin{array}{l}\text { Cita el trabajo ajeno } \\
\text { sistemáticamente y } \\
\text { diferencia su aportación }\end{array}$ \\
\hline Resultados & $\begin{array}{l}\text { No se ha resuelto el } \\
\text { problema planteado o la } \\
\text { solución es errónea. }\end{array}$ & $\begin{array}{l}\text { Muestra errores y } \\
\text { omisiones y no se ajustan } \\
\text { a la metodología. }\end{array}$ & $\begin{array}{l}\text { Algunos errores u } \\
\text { omisiones que no } \\
\text { comprometen las } \\
\text { conclusiones. }\end{array}$ & $\begin{array}{l}\text { Adecuados al objetivo y } \\
\text { coherentes con la } \\
\text { metodología. }\end{array}$ \\
\hline $\begin{array}{l}\text { Evaluación de la solución } \\
\text { aportada para alcanzar los } \\
\text { objetivos del trabajo }\end{array}$ & No valora el resultado & $\begin{array}{l}\text { Analiza el resultado sin } \\
\text { valorarlo }\end{array}$ & $\begin{array}{l}\text { Analiza el resultado y lo } \\
\text { valora sin validarlo }\end{array}$ & $\begin{array}{l}\text { Analiza el resultado, lo } \\
\text { valora y lo valida }\end{array}$ \\
\hline $\begin{array}{l}\text { Organización de la información } \\
\text { presentada durante la defensa }\end{array}$ & $\begin{array}{l}\text { La presentación está } \\
\text { desordenada y sin } \\
\text { estructura lógica }\end{array}$ & $\begin{array}{l}\text { La presentación está } \\
\text { estructurada de forma } \\
\text { confusa }\end{array}$ & $\begin{array}{l}\text { La presentación está } \\
\text { estructurada de forma } \\
\text { clara, con algunos fallos } \\
\text { menores }\end{array}$ & $\begin{array}{l}\text { La presentación está } \\
\text { estructurada de forma } \\
\text { clara, lógica y bien } \\
\text { cohesionada }\end{array}$ \\
\hline $\begin{array}{l}\text { Estilo, lenguaje corporal y } \\
\text { registro ajustado a la defensa } \\
\text { ante un tribunal académico }\end{array}$ & $\begin{array}{l}\text { El nivel y registro no se } \\
\text { ajusta al destinatario }\end{array}$ & $\begin{array}{l}\text { Con frecuencia no se } \\
\text { tiene en cuenta el } \\
\text { destinatario }\end{array}$ & $\begin{array}{l}\text { En general el estilo es } \\
\text { adecuado }\end{array}$ & El estilo es adecuado \\
\hline $\begin{array}{l}\text { Recursos gráficos y medios para } \\
\text { comunicar de forma efectiva. }\end{array}$ & \begin{tabular}{|l|} 
No utiliza recursos \\
gráficos ni ayudas visuales
\end{tabular} & $\begin{array}{l}\text { Utiliza medios de apoyo } \\
\text { que no ayudan }\end{array}$ & $\begin{array}{l}\text { Utiliza medios de apoyo y } \\
\text { recursos gráficos que } \\
\text { ayudan pero no mejoran } \\
\text { la efectividad }\end{array}$ & $\begin{array}{l}\text { Diseño y utilización de } \\
\text { recursos gráficos } \\
\text { excelente y equilibrada. }\end{array}$ \\
\hline $\begin{array}{l}\text { Gestión del tiempo de la } \\
\text { presentación. }\end{array}$ & $\begin{array}{l}\begin{array}{l}\text { Se excede el tiempo y el } \\
\text { tribunal interrumpe }\end{array} \\
\end{array}$ & $\begin{array}{l}\text { Se corta sin el cierre } \\
\text { adecuado- }\end{array}$ & $\begin{array}{l}\text { Demasiado deprisa o } \\
\text { demasiado lento }\end{array}$ & $\begin{array}{l}\text { Se ajusta al tiempo } \\
\text { previsto }\end{array}$ \\
\hline $\begin{array}{l}\text { Interacción con los miembros del } \\
\text { tribunal }\end{array}$ & $\begin{array}{l}\text { No contesta o responde } \\
\text { algo que no se } \\
\text { corresponde con lo } \\
\text { preguntado. }\end{array}$ & $\begin{array}{l}\text { No es capaz de contestar } \\
\text { a las preguntas }\end{array}$ & $\begin{array}{l}\text { Responde pero en algunos } \\
\text { casos, parece no entender } \\
\text { la pregunta. }\end{array}$ & $\begin{array}{l}\text { Responde de manera clara } \\
\text { y concisa a las preguntas. }\end{array}$ \\
\hline Resumen In & & & & \\
\hline & & & 78. & \\
\hline
\end{tabular}

Fig.1 Versión inicial de la rúbrica para la evaluación de los TFGs.

(c) EY-NC-ND 2017, Universitat Politècnica de València 
Mercedes Verdeguer Sancho, Juan Antonio Llorens Molina, Francisco García Breijo, Josefa Roselló Caselles, Alberto García Prats, Constanza Rubio Michavila, María Vargas Colás, María Pilar Santamarina Siurana

\section{Resultados}

Se ha conseguido elaborar una rúbrica con diferentes criterios para la valoración de los TFGs, que lleva asociada un intervalo de calificaciones para cada indicador, lo que permite realizar una evaluación objetiva. Los indicadores se han seleccionado de manera que es posible evaluar tanto el documento de TFG como la exposición realizada por el alumno durante la defensa pública del mismo.

Se han establecido cinco indicadores para evaluar el documento y otros cinco indicadores para evaluar la exposición del mismo. Asímismo, para cada indicador se han definido cuatro niveles de desarrollo, y cada nivel lleva asociada una puntuación númerica, para que la valoración del TFG se ciña a criterios objetivos y medibles.

Con el fin de validar la rúbrica y realizar las mejoras y correcciones oportunas, se ha preparado una encuesta (Figura 2), que se va a distribuir entre los diferentes grupos de interés (estudiantes de $4^{\circ}$ curso, profesorado y miembros de tribunales de evaluación) para que muestren su conformidad (Sí) o disconformidad con cada indicador (No) y añadan las observaciones que consideren necesarias. 


\begin{tabular}{|c|c|c|c|c|c|c|}
\hline \multicolumn{7}{|c|}{ MEMORIA } \\
\hline INDICADOR & NIVEL1 & NIVEL2 & NIVEL3 & NIVEL4 & \begin{tabular}{|l|} 
ESTOY DE \\
ACUERDO
\end{tabular} & OBSERVACIONES/SUGERENCIAS \\
\hline \begin{tabular}{|l|} 
Adecuación a \\
objetivos \\
formativos y \\
competencias \\
de la titulación
\end{tabular} & $\begin{array}{l}\text { No se adecúa } \\
\text { a los objetivos } \\
\text { formativos. }\end{array}$ & $\begin{array}{l}\text { Se adecúa } \\
\text { de forma } \\
\text { parcial. }\end{array}$ & $\begin{array}{l}\text { Se adecúa en } \\
\text { gran medida a } \\
\text { los objetivos } \\
\text { formativos. }\end{array}$ & \begin{tabular}{|l|} 
Se adecúa de \\
forma completa a \\
los objetivos \\
formativos.
\end{tabular} & $\begin{array}{l}\text { Osi O } \\
\text { No }\end{array}$ & \\
\hline \begin{tabular}{|l} 
Introducción \\
(antecedentes) \\
y Objetivos
\end{tabular} & $\begin{array}{l}\text { No cita o lo } \\
\text { hace } \\
\text { exclusivamente } \\
\text { con fuentes } \\
\text { meramente } \\
\text { divulgativas. } \\
\text { No aparecen } \\
\text { de manera } \\
\text { explicita los } \\
\text { objetivos o se } \\
\text { confunden con } \\
\text { plan de trabajo } \\
\text { o metodologia. } \\
\text { No se plantea } \\
\text { el problema } \\
\text { que da sentido } \\
\text { al TFG. }\end{array}$ & \begin{tabular}{|l|} 
Cita otros \\
trabajos, \\
pero \\
obviando \\
aspectos \\
importantes \\
del tema \\
tratado. \\
Los objetivos \\
están \\
enumerados \\
pero el \\
problema al \\
que \\
responde el \\
TFG no está \\
planteado.
\end{tabular} & $\begin{array}{l}\text { Cita otros } \\
\text { trabajos, } \\
\text { abarcando los } \\
\text { distintos } \\
\text { aspectos del } \\
\text { tema tratado. } \\
\text { Los objetivos } \\
\text { son definidos y } \\
\text { enumerados, } \\
\text { aunque de } \\
\text { forma } \\
\text { incompleta. }\end{array}$ & \begin{tabular}{|l|} 
Cita el trabajo \\
ajeno \\
sistemáticamente \\
relacionándolos \\
con los \\
contenidos del \\
TFG. \\
Los objetivos \\
están claramente \\
definidos y \\
enumerados. \\
Responden a \\
problemas \\
claramente \\
definidos.
\end{tabular} & ๑SI O & \\
\hline Resultados & $\begin{array}{l}\text { La expresión } \\
\text { de los } \\
\text { resultados es } \\
\text { claramente } \\
\text { inadecuada o } \\
\text { existen errores } \\
\text { metodológicos } \\
\text { que afectan a } \\
\text { su validez. No } \\
\text { dan respuesta } \\
\text { a los objetivos } \\
\text { planteados. }\end{array}$ & $\begin{array}{l}\text { Muestran } \\
\text { errores y } \\
\text { omisiones } \\
\text { en la } \\
\text { expresión de } \\
\text { los } \\
\text { resultados } \\
\text { (gráficos, } \\
\text { unidades, } \\
\text { etc.) o no se } \\
\text { ajustan a la } \\
\text { metodología. }\end{array}$ & $\begin{array}{l}\text { La expresión de } \\
\text { los resultados } \\
\text { es formalmente } \\
\text { correcta. } \\
\text { Se aprecian } \\
\text { errores u } \\
\text { omisiones que } \\
\text { no afectan } \\
\text { sustancialmente } \\
\text { a las } \\
\text { conclusiones. }\end{array}$ & \begin{tabular}{|l|} 
Los resultados \\
están \\
correctamente \\
expresados, \\
están bien \\
relacionados con \\
los objetivos del \\
TFG y son \\
coherentes con \\
su metodología.
\end{tabular} & $\begin{array}{l}\text { OSI O } \\
\text { NO }\end{array}$ & \\
\hline \begin{tabular}{|l} 
Evaluación de \\
la solución \\
aportada para \\
alcanzar los \\
objetivos del \\
trabajo
\end{tabular} & $\begin{array}{l}\text { No valora el } \\
\text { resultado. }\end{array}$ & $\begin{array}{l}\text { Analiza el } \\
\text { resultado sin } \\
\text { valorarlo. }\end{array}$ & $\begin{array}{l}\text { Analiza los } \\
\text { resultados y los } \\
\text { valora. }\end{array}$ & \begin{tabular}{|l|} 
Analiza los \\
resultados, los \\
valora, valida y \\
los contrasta con \\
los objetivos \\
planteados.
\end{tabular} & $\begin{array}{l}\odot S I O \\
\text { NO }\end{array}$ & \\
\hline $\begin{array}{l}\text { Adecuación } \\
\text { del documento } \\
\text { a la normativa } \\
\text { en cuanto a } \\
\text { estructuray } \\
\text { aspectos } \\
\text { formales }\end{array}$ & $\begin{array}{l}\text { Ausencia } \\
\text { injustificada de } \\
\text { alguna de las } \\
\text { secciones del } \\
\text { documento y/o } \\
\text { errores } \\
\text { importantes en } \\
\text { cuestiones } \\
\text { formales. }\end{array}$ & \begin{tabular}{|l|} 
Aparecen \\
todas las \\
secciones \\
del \\
documento \\
pero se \\
observan \\
errores \\
sistemáticos \\
en \\
cuestiones \\
formales: \\
tablas, pies \\
de figuras, \\
etc.
\end{tabular} & $\begin{array}{l}\text { Aparecen todas } \\
\text { las secciones } \\
\text { del documento } \\
\text { pero se } \\
\text { observan fallos } \\
\text { puntuales en } \\
\text { cuestiones } \\
\text { formales: } \\
\text { tablas, pies de } \\
\text { figuras, etc. }\end{array}$ & $\begin{array}{l}\text { Aparecen todas } \\
\text { las secciones del } \\
\text { documento y no } \\
\text { se observan } \\
\text { errores } \\
\text { significativos en } \\
\text { cuestiones } \\
\text { formales: tablas, } \\
\text { pies de figuras, } \\
\text { etc. }\end{array}$ & $\begin{array}{l}\text { @SI O } \\
\text { NO }\end{array}$ & \\
\hline \multicolumn{7}{|c|}{ PRESENTACIÓN } \\
\hline $\begin{array}{l}\text { Organización } \\
\text { de la } \\
\text { información } \\
\text { presentada } \\
\text { durante la } \\
\text { defensa }\end{array}$ & \begin{tabular}{|l} 
La \\
presentación \\
está \\
desordenada y \\
sin estructura \\
lógica.
\end{tabular} & \begin{tabular}{|l|} 
La \\
presentación \\
está \\
estructurada \\
de forma \\
confusa. \\
\end{tabular} & \begin{tabular}{|l|} 
La presentación \\
está \\
estructurada de \\
forma clara, con \\
algunos fallos \\
menores.
\end{tabular} & \begin{tabular}{|l|} 
La presentación \\
está estructurada \\
de forma clara, \\
logica y bien \\
cohesionada.
\end{tabular} & $\begin{array}{l}\odot S I O \\
\text { NO }\end{array}$ & \\
\hline
\end{tabular}

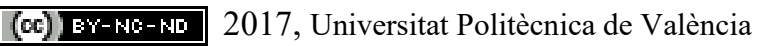


Mercedes Verdeguer Sancho, Juan Antonio Llorens Molina, Francisco García Breijo, Josefa Roselló Caselles, Alberto García Prats, Constanza Rubio Michavila, María Vargas Colás, María Pilar Santamarina Siurana

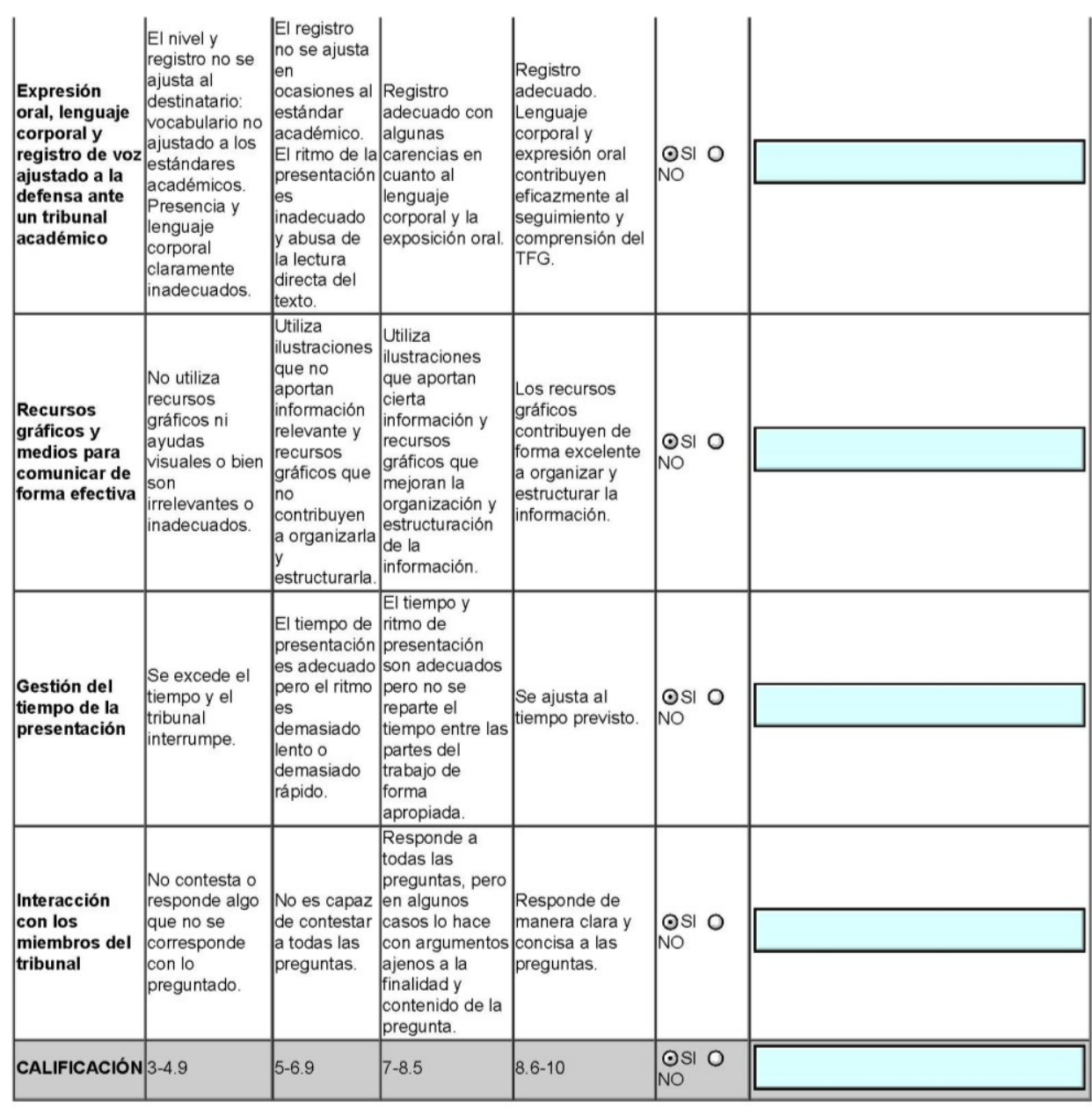

Fig. 2. Encuesta sobre la rúbrica elaborada para evaluar los TFGs.

\section{Conclusiones}

Se ha conseguido elaborar una encuesta rúbrica para valorar los TFGs en la ETSIAMN con criterios de evaluación medibles y puntuables numéricamente. Es necesario que la rúbrica sea distribuída entre los diferentes grupos de interés para que puedan expresar sus opiniones y revisar los indicadores y descriptores actuales, con el fin de validar la rúbrica y que pueda ser utilizada en las próximas convocatorias de defensa de TFGs

\section{Referencias:}

BERROCOSO, J. V. (2014). "El uso de e-rúbricas para la evaluación de competencias en estudiantes universitarios. Estudio sobre fiabilidad del instrumento". REDU. Revista de Docencia Universitaria, 12(1), 49-79.

GARCÍA-ROS, R. (2011). "Análisis y validación de una rúbrica para evaluar habilidades de presentación oral en contextos universitarios". Electronic Journal of Research in Educational Psychology, 9(3), 1043-1062. 
JAUME-I-CAPÓ, A., GUERRERO, C., MIRÓ, J., \& EGEA, A. (2012). “Elaboración de una rúbrica para la evaluación TFG y TFM de informática en la Universitat de les Illes Balears". Jornadas de Enseñanza de la Informática (18es: 2012: Ciudad Real). Disponible en http://upcommons.upc.edu/handle/2099/15089 [Consulta 30 de mayo de 2017]

MORENO, V., HERNÁNDEZ-LEO, D., CAMPS, I., MELERO, J. (2012). "Uso de rúbricas para el seguimiento y evaluación de los trabajos de fin de grado". En: II Congreso Internacional sobre evaluación por competencias mediante eRúbricas (24-26 octubre 2012 Málaga). Disponible en https://repositori.upf.edu/handle/10230/19801 [Consulta 30 de mayo de 2017]

TORRES GORDILLO, J. J., PERERA RODRÍGUEZ, V. H. (2010). "La rúbrica como instrumento pedagógico para la tutorización y evaluación de los aprendizajes en el foro online en educación superior". Pixel-Bit, 36, 141-149.

RAPOSO, M., MARTÍNEZ, E. (2011). "La rúbrica en la enseñanza universitaria: un recurso para la tutoría de grupos de estudiantes". Formación universitaria, 4(4), 19-28.

VALDERRAMA VALLÉS, E., RULLÁN AYZA, M., SÁNCHEZ CARRACEDO, F., PONS, J., CORES PRADO, M. F., BISBAL RIERA, J. (2009). "La evaluación de competencias en los Trabajos Fin de Estudios". XV JENUI. Barcelona, 405-412.

WEBSTER, F., PEPPER, D., JENKINS, A. (2000). "Assessing the undergraduate dissertation". Assessment and Evaluation in Higher Education, 25(1), 71-80. doi:10.1080/02602930050025042 